\title{
Estado nutricional e teor de glicosídeos cianogênicos em plantas de seringueira
}

\author{
Larissa Alexandra Cardoso Moraes ( $\left.{ }^{*}\right)$; Adônis Moreira ( $\left.{ }^{1}\right)$; Siu Mui Tsai $\left({ }^{2}\right)$ \\ (1) Embrapa Soja, Caixa Postal 238, 86001-970 Londrina (PR). \\ (2) Centro de Energia Nuclear na Agricultura, Caixa Postal 96, 13400-970 Piracicaba (SP). \\ (*) Autora correspondente: larissa.moraes@cnpso.embrapa.br
}

Recebido: 30/out./2009; Aceito: 25/set./2010.

\begin{abstract}
Resumo
A presença de altos teores de glicosídeos cianogênicos pode predispor a planta ao secamento de painel. O objetivo deste trabalho foi verificar a influência do estado nutricional na formação dos glicosídeos cianogênicos na seringueira, utilizando dois experimentos: a) jardim clonal com copa de híbridos de Hevea pauciflora e H. guianensis var. marginata (CPAA C 01) e de $\mathrm{H}$. rigidifolia (CPAA C 81); e b) viveiro com H. brasiliensis. No jardim clonal foram coletadas ao acaso, folhas de 23 plantas, enquanto no viveiro, foi realizado experimento em delineamento inteiramente casualisado com cinco repetições, com tratamentos em esquema fatorial 2 × 4, com duas doses de nitrogênio - N (0,8 e 1,6\%, fonte: uréia) e quatro de manganês - Mn $\left(0,10,50\right.$ e $\left.100 \mathrm{mg} \mathrm{kg}^{-1}\right)$. Na seringueira, independentemente da idade e da espécie de Hevea, os teores foliares de N, Mn e clorofila tiveram correlação positiva com o HCNp das folhas novas e diagnóstico, podendo assim serem utilizados em futuros processos de seleção. Em concentrações baixas de N (0,8\%), as doses de Mn influenciaram a absorção de N, P, K, Ca, S, Fe e Mn pelas plantas de seringueira (Hevea brasiliensis), na fase de viveiro.
\end{abstract}

Palavras-chave: Hevea spp., glicosídeo cianogênico, nutrição mineral, clorofila.

\section{Nutritional status and glycoside cyanogenic concentration in rubber tree plants}

\begin{abstract}
The presence of high cyanogenic glycosides concentrations may predispose plant to the tapping panel dryness (TPD). The aim of this work was to evaluate the effect of nutritional status in formation of cyanogenic glycosides in rubber tree. Two experiments were carried out: a) clonal nursery with crow clones hybrids of Hevea pauciflora $\times \mathrm{H}$. guianensis var. Marginata (CPAA C 01) and $\mathrm{H}$. rigidifolia (CPAA C 81); and b) nursery with $\mathrm{H}$. brasiliensis. In clonal nursery, twenty three foliar samples were collected, while in nursery, the experiment was carried out using a completely randomized design in factorial scheme 2 x 4 with five replicates. Two nitrogen doses (0.8 and 1.6\%, urea as source) and four manganese doses (0, 10, 50 and 100 mg $\mathrm{kg}^{-1}, \mathrm{MnSO}_{4}$ as source) were used. Regardless of age and species of Hevea, only the N, chlorophyll and Mn concentrations showed relationship with the HCNp in young leaves. These variables should be used in future selection processes. At low $\mathrm{N}$ $(0.8 \%)$ concentration, the Mn doses influenced the N, P, K, Ca, S, Fe and Mn uptake by rubber tree (Hevea brasiliensis) in the nursery condition.
\end{abstract}

Key words: Hevea spp., cyanogenic glycosides, mineral nutrition, chlorophyll.

\section{INTRODUÇÃO}

O gênero Hevea possui amplo grau de dispersão, estando presente em praticamente toda a Amazônia Legal (Moraes, 1977). Das onze espécies do gênero atualmente catalogadas, a Hevea brasiliensis foi a de maior capacidade produtiva e representa $99 \%$ da borracha natural produzida no Mundo, aliada à melhor qualidade do látex (GoldTHORPe e TAN, 1996).

As espécies de Hevea pertencem ao grupo de mais de 3.000 espécies que são cianogênicas e tem os mesmos glicosídeos observados em plantas de mandioca (Manihot esculenta Crantz), denominados de linamari- na, constatada em maior quantidade, e de lotaustralina (Lieberei, 2007). Os glicosídeos são armazenados nos vacúolos das células e, por injúria mecânica, são postos em contato com a linamarase, uma $\beta$-glicosidase náo específica encontrada no apoplasto, a qual quebra a linamarina em glicose e acetocianohidrina que, por sua vez, é quebrada em HCN e carbonila pela atividade da $\alpha$-hidroxinitrilase (Lieberei, 2007). Na planta, a função mais conhecida do $\mathrm{HCN}$ é a proteção contra herbívoros e no estoque de $\mathrm{N}$ e de glicose (KongsawaDWORAKUl et al., 2009).

Pesquisa recente relata que a reação de conversão dos aminoácidos L-valina e L-isoleucina em aglicona, 
passo inicial da biossíntese dos dois glicosídeos cianogênicos da mandioca (linamarina e lotaustralina), é catalisada por citocromos multifuncionais, enzimas que se encontram ligadas à membrana celular e são denominados citocromos P-450. Em seguida, ocorre a conversão de aglicona em glicosídeo cianogênico $(\mathrm{HCNp})$ pela ação da uridina 5'-difosfoglicose-glucosil transferase (ANDERSEN et al., 2000).

Processos semelhantes também ocorrem em Sorghum bicolor L. (Moench), com o envolvimento dos citocromos P-450 na formaçáo do glicosídeo cianogênico durrina a partir do aminoácido L-tirosina (SibBESEN et al., 1995). BusK e MöLler (2002) estudaram o efeito da adubação na síntese da durrina e na atividade dos citocromos P-450 em mudas de sorgo. Segundo os autores, a formação de aglicona a partir de L-tirosina catalizada pela ação dos citocromos P-450 é o fator limitante nesse processo. $\mathrm{O}$ magnésio $(\mathrm{Mg})$, potássio $(\mathrm{K})$, cobre $(\mathrm{Cu})$, ferro $(\mathrm{Fe})$, manganês $(\mathrm{Mn})$ e zinco $(\mathrm{Zn})$ atuam, do mesmo modo, como ativadores enzimáticos e cofatores de reaçôes enzimáticas que, se presentes nas plantas em deficiência ou excesso, podem interferir diretamente no metabolismo das plantas (Marschner, 1995).

A compreensão da influência das condiçôes de crescimento da planta na formação do glicosídeo cianogênico aliada ao potencial genético permitirá maior confiabilidade no processo de seleçáo de plantas. $\mathrm{O}$ objetivo deste trabalho foi verificar a influência do estado nutricional na formação dos glicosídeos cianogênicos em copa de híbridos de Hevea pauciflora e $H$. guianensis var. marginata (CPAA C 01) e de H. rigidifolia (CPAA C 81) e H. brasiliensis.

\section{MATERIAL E MÉTODOS}

Os experimentos foram realizados em condiçôes de campo e de viveiro, em área localizada nas coordenadas 308'S e 59 $52^{\prime} \mathrm{W}$, no município de Manaus (AM), em Latossolo Amarelo distrófico, textura argilosa $\left(730 \mathrm{~g} \mathrm{~kg}^{-1}\right.$ de argila), com os seguintes atributos químicos na camada de 0-20 cm: $\mathrm{pH}$ (água) $=4,3 ; \mathrm{N}$ total $=1,55 \mathrm{~g} \mathrm{~kg}^{-1} ; \mathrm{P}$ (Mehlich 1) $=2,0 \mathrm{mg} \mathrm{dm}^{-3} ; \mathrm{K}($ Mehlich 1$)=47,0 \mathrm{mg} \mathrm{dm}^{-3} ; \mathrm{Na}$

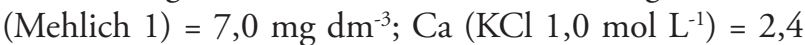

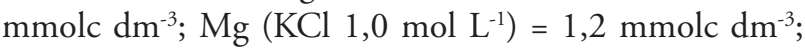
$\mathrm{Al}\left(\mathrm{KCl} 1,0 \mathrm{~mol} \mathrm{~L}^{-1}\right)=14,5$ mmolc dm${ }^{-3} ; \mathrm{H}+\mathrm{Al}=80,4$ mmolc $\mathrm{dm}^{-3}$; matéria orgânica, MO (Walkley Black) = $46,89 \mathrm{~g} \mathrm{~kg}^{-1}$; $\mathrm{Cu}$ (Mehlich 1) = 0,25 $\mathrm{mg} \mathrm{dm}^{-3}$; Fe (Mehlich 1$)=333 \mathrm{mg} \mathrm{dm}^{-3} ;$ Mn $($ Mehlich 1$)=5,15 \mathrm{mg} \mathrm{dm}^{-3}$; $\mathrm{Zn}\left(\right.$ Mehlich 1) $=0,68 \mathrm{mg} \mathrm{dm}^{-3}$ e saturação por bases $(\mathrm{V})$ $=6,3 \%$ (Embrapa, 1997).

O clima predominante na região é o tropical úmido, tipo Afi, pela classificação de Köppen, com chuvas relativamente abundantes durante o ano todo (média de 2.250 $\mathrm{mm}$ ), com alta umidade relativa do ar, sendo a quantidade sempre superior a $60 \mathrm{~mm}$, nos meses de menor precipitação, período inferior a dois meses. A temperatura média anual na região (dia e noite) é de, aproximadamente, 26 ${ }^{\circ} \mathrm{C}$ (Vieira e Santos, 1987).

Análises foliares [N, fósforo $(\mathrm{P}), \mathrm{K}$, cálcio $(\mathrm{Ca})$, magnésio $(\mathrm{Mg}), \mathrm{S}$ (enxofre), B (boro), $\mathrm{Cu}, \mathrm{Fe}, \mathrm{Mn}$ e $\mathrm{Zn}-\mathrm{MA}_{\text {- }}$ LAVOLTA et al., 1997] foram feitas em 23 folíolos jovens (estádio C - Hallé et al., 1978) da seringueira dos clones de copa CPAA C 01 - Hevea pauciflora e H. guianensis var. marginata $(\mathrm{n}=12)$ e CPAA C $81-$ H. rigidifolia $(\mathrm{n}=11)$ coletados ao acaso em diferentes jardins clonais. Os teores foliares foram correlacionados com o $\mathrm{HCNp}$ determinado de acordo com o método descrito por Lieberei (1986) e modificado por MoraEs et al. (2002).

Em ambiente protegido, pares de plântulas gêmeas com o segundo lançamento maduro foram repicados para sacos de plástico, com 4,5 L de capacidade, enchidos com terra peneirada e adubada. A adubaçáo foi feita com adição de $280 \mathrm{~g}$ de superfosfato simples (20\% de P2O5), $50 \mathrm{~g}$ de cloreto de potássio $(54 \%$ de $\mathrm{K} 2 \mathrm{O})$ e $100 \mathrm{~g}$ de calcário dolomítico (PRNT $=85 \%$ ) para cada $100 \mathrm{~L}$ de terra (Moraes et al., 2008). Os sacos com terra foram encubados por trinta dias e, após este período e antes da aplicação dos tratamentos, os seguintes resultados de atributos químicos foram obtidos: $\mathrm{pH}=4,3$; $\mathrm{N}$ total $=2,62$ $\mathrm{g} \mathrm{kg}^{-1} ; \mathrm{P}$ (Mehlich 1) $=214,0 \mathrm{mg} \mathrm{dm}{ }^{-3} ; \mathrm{K}=189,0 \mathrm{mg}$ $\mathrm{dm}^{-3} ; \mathrm{Na}=2,0 \mathrm{mg} \mathrm{dm}^{-3} ; \mathrm{Ca}=29,1 \mathrm{mmolc} \mathrm{dm}^{-3} ; \mathrm{Mg}=7,1$ mmolc dm${ }^{-3} ; \mathrm{Al}=5,1 \mathrm{mmolc} \mathrm{dm}^{-3} ; \mathrm{H}+\mathrm{Al}=10,78$ mmolc $\mathrm{dm}^{-3} ; \mathrm{MO}=52,47 \mathrm{~g} \mathrm{~kg}^{-1} ; \mathrm{Cu}=0,84 \mathrm{mg} \mathrm{dm}-3 ; \mathrm{Fe}=175$ $\mathrm{mg} \mathrm{dm}{ }^{-3} ; \mathrm{Mn}=4,34 \mathrm{mg} \mathrm{dm}^{-3} ; \mathrm{Zn}=0,84 \mathrm{mg} \mathrm{dm}^{-3} \mathrm{e} \mathrm{V}=$ 27,6 \% (EMbrapa, 1997).

No primeiro lançamento de folhas após o epicótilo com as folhas recém-maduras, foi feita uma amostragem composta com a coleta de um folíolo de todas as plantas do experimento para determinação de $\mathrm{N}, \mathrm{P}, \mathrm{K}, \mathrm{Ca}, \mathrm{Mg}$, S, B, Cu, Fe, Mn e Zn (Malavolta et al., 1997). Após três meses de transplantadas e antes da aplicação dos tratamentos (primeiro lançamento), foram retiradas amostras de folhas recém-maduras de todas as plantas gêmeas e agrupadas em oito amostras compostas para avaliação do estado nutricional, nos quais apresentaram, na média, os seguintes teores: $\mathrm{N}=29,18 \pm 2,48 \mathrm{~g} \mathrm{~kg}^{-1}, \mathrm{P}=2,07 \pm 0,20$ $\mathrm{g} \mathrm{kg}^{-1}, \mathrm{~K}=5,56 \pm 0,53 \mathrm{~g} \mathrm{~kg}^{-1}, \mathrm{Mg}=1,86 \pm 0,20 \mathrm{~g} \mathrm{~kg}^{-1}, \mathrm{~S}=$ $2,01 \pm 0,30 \mathrm{~g} \mathrm{~kg}^{-1}, \mathrm{~B}=24,47 \pm 3,34 \mathrm{mg} \mathrm{kg}^{-1}, \mathrm{Cu}=26,02$ $\pm 7,79 \mathrm{mg} \mathrm{kg}^{-1}, \mathrm{Fe}=63,41 \pm 7,53 \mathrm{mg} \mathrm{kg}^{-1}, \mathrm{Mn}=75,85$ $\pm 7,32 \mathrm{mg} \mathrm{kg}^{-1}$ e $\mathrm{Zn}=25,42 \pm 2,66 \mathrm{mg} \mathrm{kg}^{-1}$, clorofila $=$ $125,78 \pm 9,82 \mu \mathrm{g} \mathrm{cm}^{-2}$.

Os tratamentos foram dispostos em delineamento inteiramente casualizado em esquema fatorial $2 \times 4$ (duas doses de $\mathrm{N}$ x quatro doses de $\mathrm{Mn}$ ), com cinco repetiçóes. As doses de $\mathrm{N}$ foram definidas pela quantidade de aplica-

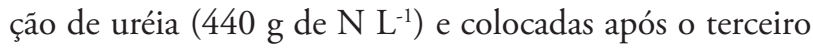
lançamento foliar, partindo-se de uma dose base de 150 $\mathrm{mL} /$ saquinho de uma solução de uréia a $0,2 \%$ de N. Assim, o tratamento A1 recebeu uma aplicação quinzenal 
da solução e o tratamento A2 duas aplicaçôes de $\mathrm{N}$ no mesmo período. Em ambos os tratamentos, as aplicaçóes das doses foram realizadas num período de dois meses, perfazendo quatro aplicaçóes no tratamento A1 $(0,8 \%$ de $\mathrm{N})$ e oito aplicaçóes no tratamento A2 (1,6\% de N). Após o terceiro lançamento foliar foram aplicadas quatro doses de $\mathrm{Mn}\left(0,10,50\right.$ e $100 \mathrm{mg} \mathrm{kg}{ }^{-1}$ - fonte: $\left.\mathrm{MnSO}_{4} \cdot \mathrm{H}_{2} \mathrm{O}\right)$.

Seis meses após o transplantio foi determinada a unidade SPAD com o clorofilômetro (Minolta 502) nos três folíolos no estágio C (HaLlÉ et al., 1978) e na folha diagnóstico - recém-madura (Malavolta, 1992), com valores convertidos em teor de clorofila $\left(\mu \mathrm{g} \mathrm{cm}^{-2}\right.$ ) (GUIMARÁES et al., 1999). Após as leituras dos folíolos, estes foram coletados para avaliação do estado nutricional $(\mathrm{N}$, $\mathrm{P}, \mathrm{K}, \mathrm{Ca}, \mathrm{Mg}, \mathrm{S}, \mathrm{B}, \mathrm{Cu}, \mathrm{Fe}, \mathrm{Mn}$ e $\mathrm{Zn}$ ), conforme método descrito por Malavolta et al. (1997). Devido os baixos teores de $\mathrm{HCNp}$ verificados na folhas diagnóstico, a determinação do teor de $\mathrm{HCNp}$ foi realizada apenas nos folíolos no estádio C.

De acordo com o delineamento proposto, os resultados foram analisados estatisticamente através da análise de variância (ANOVA), teste $\mathrm{F}$, correlaçóes e regressóes a 5 \% de significância (Pimentel Gomes e Garcia, 2002).

\section{RESULTADOS E DISCUSSÃO}

No jardim clonal, apenas os teores de $\mathrm{N}$ e $\mathrm{Mn}$ proporcionaram correlaçôes significativas com o $\mathrm{HCNp}$ (Tabela 1 ). Com relação aos teores foliares de N, P, K, Ca, $\mathrm{Mg}, \mathrm{S}, \mathrm{B}, \mathrm{Cu}, \mathrm{Fe}, \mathrm{Mn}$ e $\mathrm{Zn}$, ocorreram diferenças com os considerados adequados por Malavolta et al. (1997) para Hevea brasiliensis. Tais diferenças, possivelmente, se devem ao estádio das folhas utilizadas para análise, interferindo nos teores dos nutrientes (MARSCHNER, 1995), além das diferentes espécies de Hevea analisadas (CPAA C 01 e CPAA C 81). Segundo Moraes e MoRAES (2008), não existem faixas adequadas de nutrientes para as espécies de Hevea (H. guianensis, H. rigidifolia e
H. pauciflora) utilizadas nos cruzamentos de clones de copa. Com relação somente ao $\mathrm{Mn}$, houve significância com o teor de clorofila e HCNp (Figura 1).

A correlação observada entre o $\mathrm{N}$ na folha e o HCNp também foi observada por Gleadow e Woodrow (2000), em estudos com eucalipto (Eucalyptus cladocalyx F. Muell.), porém, tal inferência não é o esperado para outras espécies, visto que em trabalhos realizados com outra euforbiácea (mandioca) por CARDoso JúNIOR (2005) e TAlatala e Loreto (2008) náo foram constatadas tais interaçôes. $\mathrm{Na}$ seringueira, os glicosídeos cianogênicos podem desempenhar importante papel na regeneração do látex como fonte de $\mathrm{N}$ e C. Kongsawadworakul et al. (2009) relatam a redução do teor de HCNp na área de drenagem do látex após a sangria. Embora os HCNps não estejam presentes no látex, esses contém enzimas relacionadas com seu metabolismo, como a $\beta$-glicosidase e a $\beta$-cianoialanina síntase, capaz de incorporar o $\mathrm{HCN}$ liberado no metabolismo da planta via asparagina (BLUMENTHAL et al., 1968).

Busk e Möller (2002) estudaram o efeito da adubação nitrogenada na síntese da durrina bem como na atividade dos citocromos P-450 em mudas de sorgo (Sorghum bicolor L. Moench) e constataram o aumento da atividade das enzimas acompanhado pelo aumento do conteúdo de durrina na presença de $\mathrm{KNO}_{3}$, a partir da dose $10 \mathrm{mM}$. Segundo esses autores, a formação de aglicona a partir de L-tirosina, catalisada pela ação dos citocromos P-450, apresenta-se como fator limitante nesse processo de biossíntese.

No caso da correlação observada com o Mn (Tabela 1 e Figura 1), este pode estar atuando na fase inicial da biossíntese dos glicosídeos cianogênicos, que é catalisada por citocromos multifuncionais, enzimas ligadas à membrana e denominadas citocromos P-450, cujo aumento de sua concentração foi observado em plantas superiores na presença de Mn (Reichardt et al., 1980).

Em plantas que se multiplicam por enxertia, como a seringueira, os mecanismos de tolerância ao Mn são lo-

Tabela 1. Estatística descritiva dos teores foliares de N, P, K, Ca, Mg, S, B, Cu, Fe, Mn e Zn e a significância das correlações com o HCNp das folhas - clones CPAA C $01(\mathrm{n}=12)$ e CPAA C $81(\mathrm{n}=11)$

\begin{tabular}{|c|c|c|c|c|c|c|c|c|c|c|c|}
\hline \multirow[t]{2}{*}{ Variáveis } & $\mathbf{N}$ & $\mathbf{P}$ & K & $\mathrm{Ca}$ & Mg & $\mathbf{s}$ & B & $\mathrm{Cu}$ & $\mathrm{Fe}$ & Mn & Zn \\
\hline & \multicolumn{6}{|c|}{$\mathbf{g ~ k g}^{-1}$} & \multicolumn{5}{|c|}{$\mathrm{mg} \mathrm{kg}^{-1}$} \\
\hline Média & 44,6 & 4,7 & 25,7 & 1,4 & 2,5 & 2,0 & 22,9 & 10,9 & 103,9 & 43,1 & 70,0 \\
\hline Mediana & 45,2 & 5,0 & 25,2 & 1,4 & 2,7 & 2,1 & 23,9 & 9,9 & 106,6 & 40,3 & 77,0 \\
\hline Mínimo & 28,5 & 5,2 & 19,6 & 1,1 & 1,6 & 1,6 & 14,5 & 7,0 & 78,3 & 21,7 & 21,0 \\
\hline Máximo & 60,5 & 5,5 & 31,0 & 1,8 & 3,1 & 2,6 & 30,5 & 14,5 & 128,3 & 86,9 & 122,0 \\
\hline Desvio-padrão & 9,1 & 0,9 & 4,0 & 0,3 & 0,5 & 0,4 & 5,2 & 2,9 & 18,3 & 16,0 & 31,7 \\
\hline C.V. (\%) & 20,3 & 18,5 & 15,6 & 18,5 & 21,8 & 15,2 & 22,8 & 26,4 & 17,6 & 37,1 & 45,3 \\
\hline HCNp & * & NS & NS & NS & NS & NS & NS & NS & NS & * & NS \\
\hline Teores ${ }^{(1)}$ & $26,0-35,0$ & $1,6-2,3$ & $10-14$ & $7,6-8,2$ & $1,7-2,4$ & $1,8-2,6$ & $20-70$ & $10-15$ & $70-90$ & $15-40$ & $20-30$ \\
\hline
\end{tabular}

* $\mathrm{e}^{\mathrm{NS}}=$ Significativo e não significativo a $5 \%$ de probabilidade respectivamente. ${ }^{(1)}$ Teores considerados adequados por Malavolta et al. (1997) - H. brasiliensis. 


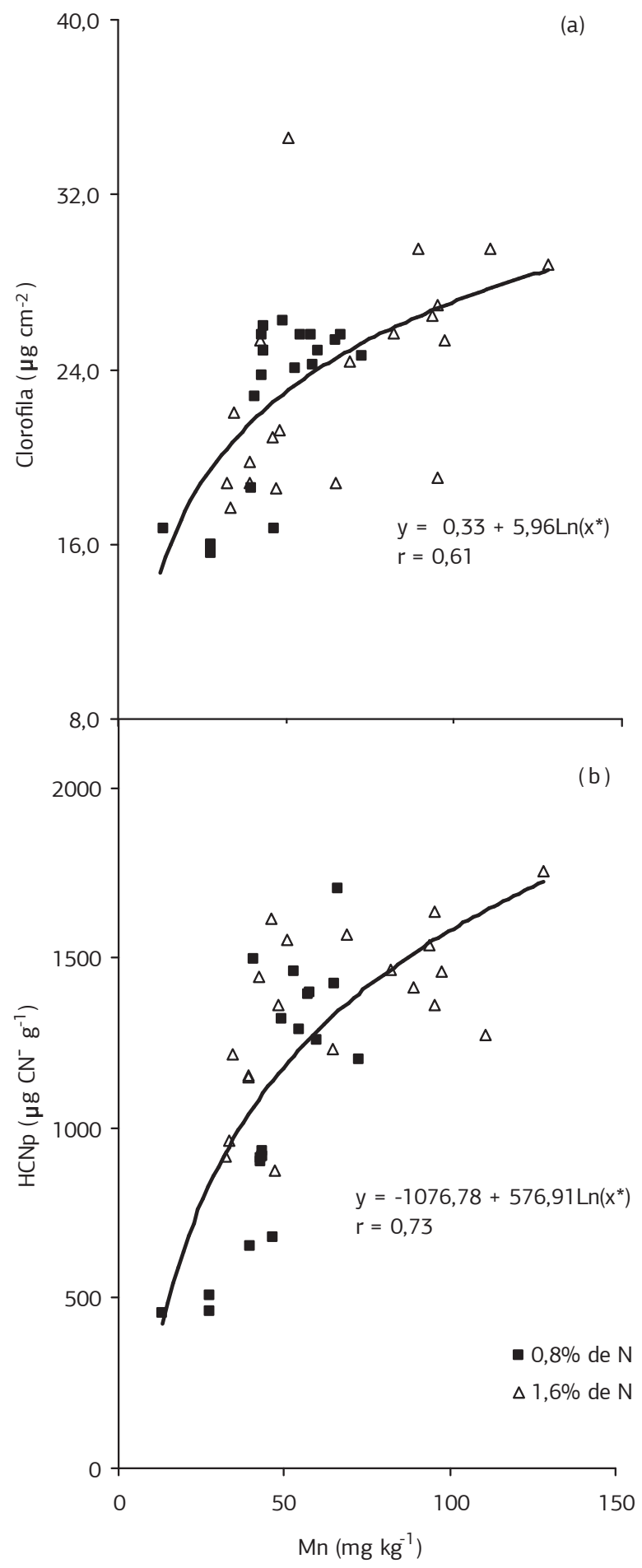

Figura 1. Correlaçôes dos teores de Mn com os teores de clorofila (a) e de $\mathrm{HCNp}$ (b), em folhas novas de seringueira (H. brasiliensis), para cada dose de N. (*significativo a 5\% de probabilidade).

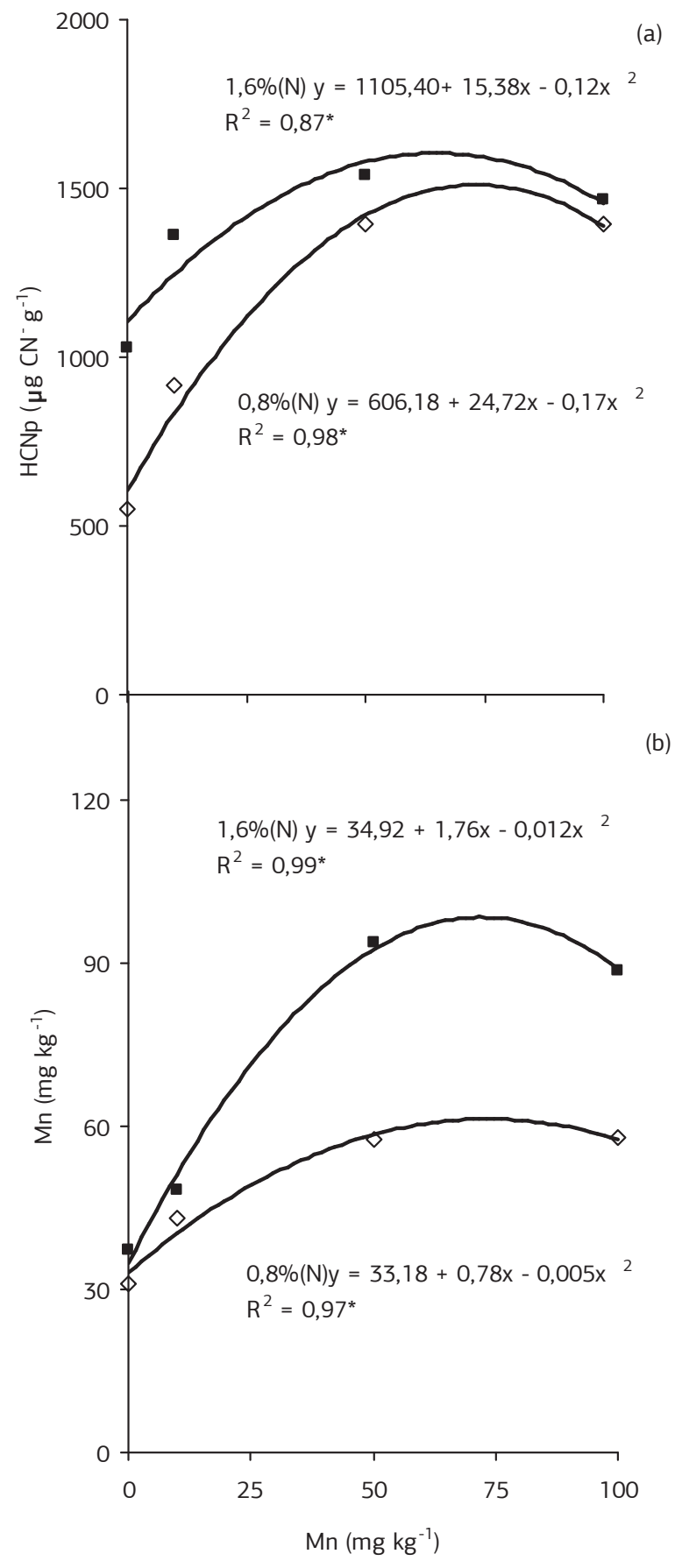

Figura 2. Efeito das doses de Mn com as de N (0,8 e 1,6\% de uréia) sobre o teor de $\mathrm{HCNp}$ (a) e Mn (b), em folhas novas de seringueira (H. brasiliensis). (*significativo a 5\% de probabilidade. Cada ponto representa a média de cinco repetiçóes).

Tabela 2. Análise de variância e valores do teste F sobre o efeito da aplicação de nitrogênio e manganês nos teores de N, Mn, clorofila e $\mathrm{HCNp}$ em folhas jovens de mudas de seringueira

\begin{tabular}{lccccc|} 
Variável & Grau de liberdade & N & Mn & Clorofila & HCNp \\
\hline Nitrogênio (a) & 1 & $7,93^{*}$ & $20,74^{* *}$ & $0,91^{\text {NS }}$ & $41,77^{* *}$ \\
Manganês (b) & 3 & $2,49^{\text {Ns }}$ & $22,82^{* *}$ & $23,38^{* *}$ & $51,35^{* *}$ \\
\hline a x b & 3 & $4,69^{*}$ & $3,62^{*}$ & $4,21^{*}$ & $5,45^{*}$
\end{tabular}

$* *, * \mathrm{e}^{\mathrm{NS}}=$ Significativo a $1 \%$ e $5 \%$ de probabilidade e năo significativo respectivamente. 
calizados nos ramos e governados pela interação portaenxerto $\mathrm{x}$ enxerto de genótipos tolerantes e náo tolerantes, sendo o genótipo do enxerto quem determina a tolerância da planta a altas concentraçôes desse nutriente no substrato e nas folhas (MARSCHNER, 1995). A julgar pelas caracterizaçóes visuais dos efeitos tóxicos do $\mathrm{Mn}$ descritas por Foy (1976), as concentraçōes $\left(43,1 \pm 16,0 \mathrm{mg} \mathrm{kg}^{-1}\right)$ verificadas nas plantas de jardim clonal não determinaram injúria nas plantas.

As interaçôes entre as doses de $\mathrm{N}$ e $\mathrm{Mn}$ com os teores foliares, clorofila e $\mathrm{HCNp}$ encontram-se na Tabela 2 e na Figura 2. A análise de variância demonstra significância nos teores de N, Mn e HCNp nas folhas jovens com aumento dos teores de $\mathrm{N}$, em funçáo das doses de $\mathrm{N}$; o mesmo foi observado com os teores de $\mathrm{Mn}$, havendo neste caso, interação entre esses dois tratamentos (Tabela 2). O teor de clorofila também teve influência das doses de $\mathrm{N}$ e $\mathrm{Mn}$ corroborando os resultados observados no experimento de jardim clonal (Tabela 2 e Figura 1).

Similar ao observado com os clones de copa CPAA C 01 e CPAA C 81, as plantas de H. brasiliensis também tiveram correlação significativa entre os teores de $\mathrm{N}$ e $\mathrm{Mn}$ com o HCNp em folhas jovens (Figura 2). Altos teores de glicosídeos cianogênicos podem predispor as plantas à "seca de painel", uma enfermidade fisiológica quando em regime de sangria (NANDris et al., 2004), e causar efeito depressivo na produçáo das copas enxertadas de seringueira (Moraes et al., 2002). Conclui-se, portanto, que o monitoramento do estado nutricional, em especial do $\mathrm{N}$ e Mn, podem ser uma ferramenta importante no processo de seleçáo de clones de copa.
Nos teores de HCNp também houve correlação significativa com os teores de $\mathrm{N}$ e $\mathrm{Mn}$ nas folhas diagnóstico [ $\hat{\mathrm{y}}$ $=21,465+0,006 x, r=0,54$ e $\hat{y}=19,025+0,076 x, r=0,61$ $(\mathrm{p}<0,05)]$. Com os demais nutrientes não ocorreu interação com o $\mathrm{HCNp}$ da planta. Esses resultados indicam a possibilidade de se utilizar os teores de $\mathrm{N}$ e $\mathrm{Mn}$ da folha diagnóstico no monitoramento dos teores de $\mathrm{HCNp}$, fato que pode contribuir nos trabalhos futuros sobre seca do painel e na incompatibilidade de combinaçóes de clones nos estudos de enxertia de copa.

Verificou-se que as mudas de Hevea brasiliensis tiveram, na média, altos teores de HCNp nas folhas antes do início do experimento $(\mathrm{HCNp}=719,22 \pm 130,26 \mu \mathrm{g}$ de $\left.\mathrm{CN}^{-} \mathrm{g}^{-1}\right)$ e 60 dias após a aplicação das doses de $\mathrm{N}$ e $\mathrm{Mn}$ $\left(0,8 \%\right.$ de $\mathrm{N}=1063,05 \pm 380,61 \mu \mathrm{g}$ de $\mathrm{CN}^{-} \mathrm{g}^{-1}$ e $1,6 \%$ de $\left.\mathrm{N}=1347,23 \pm 247,15 \mu \mathrm{g} \mathrm{de} \mathrm{CN}^{-} \mathrm{g}^{-1}\right)$.

$\mathrm{Na}$ dose de $0,8 \%$ de $\mathrm{N}$, a aplicação de $\mathrm{Mn}$ influenciou os teores de N, P, K, Ca, S, Cu, Fe e Mn, enquanto na $1,6 \%$ de $\mathrm{N}$, somente o teor de Mn apresentou significância (Tabela 3). Não foram observados sintomas visuais de deficiência ou toxidez de $\mathrm{N}$ ou $\mathrm{Mn}$ nas plantas de seringueira. O Mn participa como constituinte ou ativador enzimático, e sua absorção e teor podem ser influenciados pela competição interiônica na presença de $\mathrm{Ca}, \mathrm{Mg}, \mathrm{Cu}$, Fe e Zn (Clarkson, 1988) ou em processos metabólicos, como a fotossíntese, respiração e a síntese de proteínas (Marschner, 1995).

A ausência de significância na dose de 1,6\% indica que as alteraçóes nos teores dos nutrientes analisados não foram dependentes da adubação nitrogenada. $\mathrm{Na}$ folha-diagnóstico, na média dos tratamentos, foram constatados os seguintes teores: $\mathrm{N}=28,73 \pm 3,88$

Tabela 3. Teores foliares de N, P, K, Ca, Mg, S, B, Cu, Fe, Mn e Zn em função das doses de N e Mn - experimento com H. brasiliensis - viveiro

\begin{tabular}{|c|c|c|c|c|c|c|c|c|c|c|c|c|}
\hline Doses & & $\mathbf{N}$ & $\mathbf{P}$ & K & $\mathrm{Ca}$ & Mg & 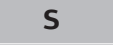 & B & $\mathrm{Cu}$ & $\mathrm{Fe}$ & Mn & $\mathrm{Zn}$ \\
\hline \multirow[t]{2}{*}{ N \% } & $\begin{array}{c}\text { Mn } \\
\text { mg kg }^{-1}\end{array}$ & \multicolumn{8}{|c|}{$\mathrm{g} \mathrm{kg}^{-1}$} & \multicolumn{3}{|l|}{$\mathrm{mg} \mathrm{kg}^{-1}$} \\
\hline & 0 & 32,65 & 3,94 & 14,64 & 3,67 & 2,47 & 2,29 & 39,74 & 9,95 & 53,06 & 31,11 & 49,08 \\
\hline \multirow[t]{3}{*}{0,8} & 10 & 37,74 & 4,18 & 14,33 & 3,12 & 3,02 & 2,07 & 49,25 & 10,24 & 38,21 & 43,20 & 50,17 \\
\hline & 50 & 40,79 & 5,12 & 17,20 & 3,82 & 2,71 & 2,53 & 47,32 & 12,94 & 52,34 & 57,44 & 59,10 \\
\hline & 100 & 39,79 & 4,91 & 16,11 & 2,15 & 2,65 & 2,84 & 32,48 & 12,36 & 79,04 & 57,82 & 54,31 \\
\hline$\beta 0$ & & 35,44 & 4,13 & 14,77 & 3,66 & 2,74 & 2,26 & 46,36 & 10,31 & 42,78 & 37,69 & 50,71 \\
\hline$\beta 1$ & & $0,058^{*}$ & $0,010^{*}$ & $0,020^{*}$ & $-0,012^{*}$ & $-0,001^{\mathrm{NS}}$ & $0,007^{*}$ & $-0,104^{\mathrm{NS}}$ & $0,027^{*}$ & $0,322^{* *}$ & $0,243^{* *}$ & $0,061^{\mathrm{NS}}$ \\
\hline \multirow[t]{2}{*}{$\mathrm{R}^{2}$} & & 0,269 & 0,290 & 0,263 & 0,342 & 0,004 & 0,24 & 0,095 & 0214 & 0,401 & 0,474 & 0,074 \\
\hline & 0 & 44,78 & 4,83 & 17,96 & 2,43 & 3,00 & 2,01 & 39,81 & 18,82 & 124,42 & 37,23 & 50,19 \\
\hline \multirow[t]{3}{*}{1,6} & 10 & 36,85 & 4,15 & 16,26 & 2,74 & 2,76 & 2,23 & 40,71 & 11,29 & 100,39 & 48,11 & 57,24 \\
\hline & 50 & 40,16 & 4,37 & 15,18 & 4,88 & 3,12 & 2,27 & 36,02 & 14,35 & 118,59 & 93,64 & 53,71 \\
\hline & 100 & 45,16 & 4,83 & 15,35 & 3,23 & 2,93 & 2,68 & 31,16 & 16,06 & 73,27 & 88,71 & 60,04 \\
\hline$\beta 0$ & & 40,35 & 4,44 & 17,05 & 2,91 & 2,93 & 2,06 & 40,75 & 15,030 & 119,69 & 45,46 & 52,62 \\
\hline$\beta 1$ & & $0,035^{\mathrm{NS}}$ & $0,003^{\mathrm{NS}}$ & $-0,022^{\mathrm{NS}}$ & $0,010^{\mathrm{NS}}$ & $0,001^{\mathrm{NS}}$ & $0,006^{\mathrm{NS}}$ & $-0,096^{\mathrm{NS}}$ & $0,003^{N S}$ & $-0,388^{\mathrm{NS}}$ & $0,537^{* *}$ & $0,067^{\text {NS }}$ \\
\hline $\mathrm{R}^{2}$ & & 0,053 & 0,023 & 0,189 & 0,130 & 0,002 & 0,161 & 0,132 & 0,001 & 0,078 & 0,568 & 0,055 \\
\hline
\end{tabular}

$* *, * \mathrm{e}^{\mathrm{NS}}=$ Significativo a $1 \%$ e $5 \%$ de probabilidade e năo significativo respectivamente. 
$\mathrm{g} \mathrm{kg}^{-1}, \mathrm{P}=1,76 \pm 0,29 \mathrm{~g} \mathrm{~kg}^{-1}, \mathrm{~K}=6,99 \pm 0,98 \mathrm{~g} \mathrm{~kg}^{-1}$, $\mathrm{Ca}=7,80 \pm 1,98 \mathrm{~g} \mathrm{~kg}^{-1}, \mathrm{Mg}=2,28 \pm 0,42 \mathrm{~g} \mathrm{~kg}^{-1}, \mathrm{~S}=$ $1,87 \pm 0,53 \mathrm{~g} \mathrm{~kg}^{-1}, \mathrm{~B}=48,85 \pm 10,06 \mathrm{mg} \mathrm{kg}{ }^{-1}, \mathrm{Cu}=$ $18,34 \pm 5,27 \mathrm{mg} \mathrm{kg}^{-1}, \mathrm{Fe}=80,60 \pm 34,10 \mathrm{mg} \mathrm{kg}^{-1}, \mathrm{Mn}$ $=104,56 \pm 40,59 \mathrm{mg} \mathrm{kg}^{-1}-\hat{\mathrm{y}}=49,821+0,619^{*} \mathrm{x}, \mathrm{R}^{2}=$ $0,47(\mathrm{p} \leq 0,05), \mathrm{Zn}=23,10 \pm 6,53 \mathrm{mg} \mathrm{kg}^{-1}$ e clorofila $=150,84 \pm 41,45 \mu \mathrm{g} \mathrm{cm}^{-2}$. Exceto o teor de clorofila, cuja faixa adequada ainda não foi definida para a seringueira, os teores foliares de $\mathrm{N}, \mathrm{P}, \mathrm{K}, \mathrm{Ca}, \mathrm{Mg}, \mathrm{S}, \mathrm{Cu}$, $\mathrm{Fe}, \mathrm{Mn}$ e $\mathrm{Zn}$, obtidos nas doses de $\mathrm{N}$ e $\mathrm{Mn}$, ficaram próximos ou dentro das faixas consideradas adequadas por Malavolta et al. (1997), para plantas adultas, e por Moreira et al. (2006) para plantas em idade de viveiro, à exceção do $\mathrm{Mn}$.

$\mathrm{Na}$ Amazônia, os solos são caracterizados pela acidez elevada, baixa fertilidade e altos teores de Al trocável (Moreira e Fageria, 2009). Bueno et al. (1989), em solução nutritiva para avaliar o efeito do Al no acúmulo de $\mathrm{Mn}$ em seringueira (Hevea brasiliensis), verificaram aumento do teor de $\mathrm{Mn}$ em todos as doses de $\mathrm{Al}$, enquanto o acúmulo foi afetado a partir de $20 \mathrm{mg} \mathrm{kg}^{-1} \mathrm{de}$ $\mathrm{Al}$ na solução. Os resultados de Bueno et al. (1989) não podem ser extrapolados para as outras espécies de $\mathrm{He}$ $v e a$, visto que em Belém, foi verificado que híbridos de H. pauciflora são sensíveis à deficiência de Mn (VIÉGAS et al., 2000). O teor de $\mathrm{Mn}$ nas folhas deficientes de $H$. pauciflora foi de $21 \mathrm{mg} \mathrm{kg}^{-1}$, valor abaixo do verificado no tratamento sem adição de $\mathrm{Mn}$ com $0,8 \%$ de N (Tabela 3). Segundo VIÉGAS et al. (2000), na Amazônia, os teores médios de $\mathrm{Mn}$ em folhas normais recém-maduras de plantas em produção ou em crescimento estáo na ordem de 68,0 $\mathrm{mg} \mathrm{kg}^{-1}$ a $311,5 \mathrm{mg} \mathrm{kg}^{-1}$; para Moreira et al. (2006) os teores foram de $193,0 \mathrm{mg} \mathrm{kg}^{-1}$ a 240,0 $\mathrm{mg} \mathrm{kg}^{-1}$ para mudas de seringueira $(H$. brasiliensis) na fase de viveiro.

\section{CONCLUSÃO}

$\mathrm{Na}$ seringueira, independentemente da idade e da espécie de Hevea, os teores foliares de nitrogênio $(\mathrm{N})$, manganês $(\mathrm{Mn})$ e clorofila têm correlação positiva com o $\mathrm{HCNp}$ das folhas novas e folha diagnóstico, permitindo o uso em futuros processos de seleção. Em concentrações baixas de $\mathrm{N}(0,8 \%)$, o Mn influencia a absorção de $\mathrm{N}, \mathrm{K}, \mathrm{P}, \mathrm{Ca}, \mathrm{S}$, Fe e Mn pelas plantas de seringueira (Hevea brasiliensis), na fase de viveiro.

\section{AGRADECIMENTOS}

Ao Pesquisador Vicente Haroldo de Figueiredo Moraes (In Memoriam) e ao Professor Euripedes Malavolta (In Memoriam) por todo ensinamento e obstinação pela pesquisa.

\section{REFERÊNCIAS}

ANDERSEN M.D.; BUSK, P.K.; SVEBDSEN, I.; MOLLER, B.L. Cytochromes P-450 from cassava (Manihot sculenta Crantz) catalyzing the first steps in the biosynthesis of the cyanogenic glucosides linamarin and lotaustralin. The Journal of Biological Chemistry, v.275, p.1966-1975, 2000.

BLUMENTHAL, S.G.; HENDRICKSON, H.R.; ABROL, Y.P.; CONN, E.E. Cyanide metabolism in higher plants. III. The biosynthesis of $\beta$-cyanoalanine. The Journal of Biological Chemistry, v.243, p.5302-5307, 1968.

BUENO, N.; HAAG, H.P.; PEREIRA, J.P.; VIÉGAS, I.J.M. Nutriçáo mineral de seringueira (Hevea spp). X. Quantidade de Al no substrato afetando a concentraçáo e o acúmulo de $\mathrm{Fe}, \mathrm{Mn}$ e Zn. Anais da Escola Superior de Agricultura Luiz de Queiroz, v.46, p.275-293, 1989.

BUSK, P.K.; MÖLLER, B.L. Dhurrin synthesis in sorghum is regulated at the transcriptional level and induced by nitrogen fertilization in older plants. Plant Physiology, v.129, p.12221231, 2002.

CARDOSO JÚNIOR, N.S.; VIANA, A.E.S.; MATSUMOTO, S.N.; SEDIYAMA, T.; AMARAL, C.L.F.; VIEIRA, A.J.P.; RAMOS, P.A.S. Efeito do nitrogênio sobre o teor de ácido cianídrico em plantas de mandioca. Acta Scientiarum Agronomy, v.27, p.603610, 2005.

CLARKSON, D.T. The uptake and translocation of manganese by roots. In: GHAHAM, R.D.; HANNAM, R.J.; UREN, N.C. (Eds.). Manganese in soils and plants. Dordrecht: Kluwer Academic, 1988. p.101-111.

EMBRAPA. Manual de métodos de análises de solo. Rio de Janeiro: Embrapa - CNPS, 1997. 212p.

FOY, C D. Differential aluminum and manganese tolerance of plant species and varieties in acid soils. Ciência e Cultura, v.28, p.150-155, 1976.

GLEADOW, R.M.; WOODROW, I.E. Temporal and spatial variation in cyanogenic glycosides in Eucalyptus cladocalyx. Tree Physiology, v.20, p.591-198, 2000.

GOLDTHORPE, C.C.; TAN, I.I. A review of environmental issues in natural rubber production. The planter, v.72, p.123-128, 1996

GUIMARÃES, T.G.; FONTES, P.C.R.; PEREIRA, P.R.G.; ALVAREZ VENEGAS, V.H.; MONNERAT, P.H. Teores de clorofila determinados por medidor portátil e sua relação com formas de nitrogênio em folhas de tomateiro cultivados em dois tipos de solo, Bragantia, v.58, p.209-216, 1999.

HALLÉ, F.; OLDERMAN, R.A.; TOMLINSON, P.B. Tropical trees and Forest. Berlin: Spirnger-Verlag, 1978. 441p.

KONGSAWADWORAKUL, P.; VIBOONJUN U.; ROMRUENSUKHAROM, P.; CHANTUMA, P.; RUDERMAN, S., CHRESTIN, H. The leaf, inner bark and latex cyanide potential of Hevea brasiliensis: Evidence for involvement of cyanogenic glucosides in rubber yield. Phytochemistry, v.70, p.730-739, 2009. 
LIEBEREI, R. South American leaf blight of the rubber tree (Hevea spp.): New steps in plant domestication using physiological features and molecular markers Annals of Botany, v.100, p.1125-1142, 2007.

MALAVOLTA, E. ABC da análise de solo e de folhas. São Paulo: Agronômica Ceres, 1992. 124p.

MALAVOLTA, E.; VITTI, G.C.; OLIVEIRA, S.A. Avaliação do estado nutricional das plantas: princípios e aplicaçóes. Piracicaba: Potafós, 1997. 319p.

MARSCHNER, H. Mineral nutrition on higher plants. New York: Academic Press, 1995. 889p.

MORAES, L.A.C.; MORAES, V.H.F.; MOREIRA, A. Efeito da cianogênese na incompatibilidade entre clones de copa de seringueira e o clone de painel IPA 1. Pesquisa Agropecuária Brasileira, v. 37, p.925-932, 2002.

MORAES, V.H.F. Rubber. In: ALVIM, P.T.; KOSLOWSKI, T.T. (Eds.). Ecophysiology of tropical crops. New York: Academic Press. 1977. p.315-331.

MORAES, V.H.F; MORAES, L.A.C. Desempenho de clones de copa de seringueira resistentes ao mal-das-folhas. Pesquisa Agropecuária Brasileira, v.45, p.852-858, 2008.

MOREIRA, A.; FAGERIA, N.K. Soil chemical attributes of Amazonas State, Brazil. Communications in Soil Science and Plant Analysis, v.40, p.2912-2925, 2009.

MOREIRA, A.; MORAES, V.H.F.; CASTRO. C. Fontes e doses de boro em porta-enxertos de seringueira. Pesquisa Agropecuária Brasileira, v.41, p.1291-1298, 2006.

NANDRIS, D.; PELLEGRIN, F.; MOREAU, R.; ABINA, J.; ANGUI, P.; CHRETIN, J. Etiology, epidemiology and environmental investigations as the causal factors of rubber tree (Hevea brasiliensis) bark necrosis: A physiological trunk disease caused by an accumulation of stresses. In: Rubber Research and Development Board. Tapping Panel Dryness Meeting. 2004, Kunning. Proceedings... Kunning: IRRDB, 2004. 19p.

PIMENTEL GOMES, F; GARCIA, C.H. Estatística aplicada a experimentos agronômicos e florestais. Piracicaba: FEALQ, 2002. 309p.

REICHHART, D.; SALAÜN, J.P.; BENVENISTE, I.; DURST, F. Time course of induction of cytochrome P-450, NADPHCytochome $c$ reductase, and cinnamic acid hydroxylase by phenobarbital, ethanol, herbicides, and manganese in higher plant microsomes. Plant Physiology, v.66, p.600-604, 1980.

SIBBESEN, O.; KOCH, B.; HALKIER, B.A.; MOLLER, B.L. Cytochrome P-450TYR is a multifunctional hemethiolate enzyme catalyzing the conversion of L-tyrosine to p-hydroxyphenylacetaldehyde oxume in the biosynthesis of the cyanogenic glucoside dhurriin in Sorghum bicolor (L.) Moench. The Journal of Biological Chemistry, v.270, p.3506-3511, 1995.

TALATALA, R.L.; LORETO, M.T.P. Cyanide content of cassava cultivars at different fertility levels and stages of maturity. Laguna: Department of Science and Technology of Philippines University. 2008, 2p.

VIÉGAS, I.J.M.; CARVALHO, J.G.; FRAZÃO, D.A.C. Desordens nutricionais na cultura da seringueira: critérios de diagnose para o solo e plantas e correção de deficiência. In: VIÉGAS, I.J.M.; CARVALHO, J.G. (Ed.). Seringueira: nutrição e adubação no Brasil. Brasília: Embrapa, 2000. p.123-173.

VIEIRA, L.S.; SANTOS, P.C.T.C. Amazônia: seus solos e outros recursos naturais. São Paulo: Ceres, 1987. 416p. 